Federal Reserve Bank of Minneapolis

Research Department

\title{
A Microfoundation for Incomplete Security Markets
}

\author{
Harold L. Cole and Narayana Kocherlakota*
}

Working Paper 577

Revised March 1997

\begin{abstract}
We consider a simple environment in which individuals receive income shocks that are unobservable to others and can privately store resources. We show that this ability to privately store can undercut the ability to shift resources across individuals to the extent that the efficient allocation only involves consumption smoothing over time, as opposed to insurance (consumption smoothing over states) if the rate of return on savings is not too far below the rate of time preference, or, alternatively, if the worst possible outcome is sufficiently dire. We also show that unlike environments without unobservable storage, the symmetric efficient allocation is decentralizable through a competitive asset market in which individuals trade risk-free bonds among themselves.

* We would like to thank Christophe Chamley, V.V. Chari, Ed Green and Tim Kehoe for comments and suggestions. The views expressed herein are the those of the authors and not necessarily those of the Federal Reserve Bank of Minneapolis or the Federal Reserve System.
\end{abstract}




\section{Introduction}

There is a wide body of empirical evidence that indicates that the complete markets paradigm provides an inadequate description of the cross-sectional behavior of individual consumption. Instead, a consensus view appears to be that the data on individual consumption are more consistent with the predictions of a dynamic incomplete markets model in which individuals cannot directly insure themselves against income risk but instead are only able to buy and sell risk-free bonds over time while facing a short-sales limit. ${ }^{1}$ However, the restrictions on asset trade in this type of model lack explicit micro-foundations. Instead, as in Geanakoplos (1990), it is typical to refer loosely to various factors, the most important of which are the moral hazard or adverse selection considerations arising from incomplete information. $^{2}$

Unfortunately, this loose motivation has not survived rigorous scrutiny. In particular, Green (1987) shows that the efficient allocation of consumption in an environment with unobservable individual income is considerably different from the equilibrium allocation of any dynamic incomplete markets model. ${ }^{3}$ His results have been extended by Thomas and Worrall (1990), Phelan and Townsend (1991), Atkeson and Lucas (1992), and others to

\footnotetext{
${ }^{1}$ Among others, see Zeldes (1989), Cochrane (1991), Carroll (1992), Deaton (1992), Deaton and Paxson (1994) and Hayashi, Altonji and Kotlikoff (1996). Similar negative findings have been found at more aggregate levels; for example, Backus, Kehoe and Kydland (1992) document that consumption is less correlated than output across countries.

${ }^{2}$ Other factors include non-concurrent life spans and transactions costs. Of these, only the problems arising from incomplete information seem capable of rationalizing the sparse nature of income insurance possibilities assumed within this literature. Transactions costs have a hard time explaining why there are many state contingent business contracts but so few income insurance contracts, especially since the bookkeeping costs associated with such contracts have steadily declined. The existence of non-concurrent lifespans only rules out a very limited set of trades, and does not rule out partial income insurance, given one's initial conditions.

${ }^{3}$ Green (1987) characterizes the efficient allocations in multi-period environments in which individuals have unobservable income shocks, and further shows that these efficient allocations are Pareto superior to any allocation implemented by competitive unmonitored trade of a set of securities (no matter how big or small this set might be).
} 
settings with unobservable productivity or preference shocks. ${ }^{4}$ Simply put, the lesson of this literature is that asymmetric information alone does not rationalize the use of incomplete security markets to allocate resources.

In this paper, we consider the symmetric efficient allocation of resources in an environment with two frictions. First, as in Green (1987), individual income is private information. Second, unlike Green (1987) or the subsequent literature, individuals can privately store without the knowledge of others. We show that this ability to privately store greatly undercuts the ability to shift resources across individuals. Hence an efficient allocation only involves consumption smoothing over time, as opposed to insurance (consumption smoothing over states) if the rate of return on storage is not too much less than the rate of time preference, or, alternatively, if the worst possible outcome is sufficiently dire. We use this characterization of a symmetric efficient allocation to prove that it is equivalent to the unique equilibrium allocation of a dynamic incomplete markets economy in which individuals competitively trade in non-contingent bonds at each date as in Bewley (1993), Huggett (1993) and Aiyagari (1994).

There is a simple intuition for our result. If the rate of return on storage is sufficiently high or if the worst possible outcome of individual income is sufficiently low, then it is efficient for society to store resources to help individuals against idiosyncratic risk. Because of the positive social storage, it is inefficient for any agent to be credit-constrained by his own transfer scheme, or, a fortiori, by the transfer scheme received by somebody with a lower income. (Here, we use "credit-constrained" to refer to individuals for whom the multiplier

\footnotetext{
${ }^{4}$ There is a rich literature on dynamic incentive contracts in settings with private information, but without storage or capital accumulation. Other important contributions include Spear and Srivastava (1987), Abreu, Pearce and Stacchetti (1990), Taub (1990), Green and Oh (1991), Atkeson and Lucas (1995), Aiyagari and Alvarez (1995), Phelan (1995), and Wang (1995).
} 
on the constraint guaranteeing nonnegative storage is positive.)

Hence, on the one hand, to be efficient and incentive-compatible, the net present value of the transfers received by high income individuals must be at least as large as the net present value of the transfers received by low income individuals. On the other hand, risk-sharing considerations imply that it is inefficient for high income individuals to receive more net present value than low income individuals. It follows that the net present value of all transfer schemes should be the same across all individuals (that is, zero). Given this restriction, the efficient allocation has to correspond to the equilibrium allocation.

Atkeson (1991), Marcet and Marimon (1992), and Khan and Ravikumar (1996) characterize efficient allocations in environments with dynamic accumulation of resources and private information. However, in all of their models, all payoff-relevant state variables are observable at the beginning of each period. In contrast, in our model, individual storage levels are an unobservable state variable. This difference forces us to develop a new line of attack relative to the current literature.

It is important to emphasize that, despite a superficial similarity, our model is in fact considerably different from those of Allen (1985) and Fudenberg, Holmstrom, and Milgrom (FHM) (1990). These papers characterize optimal principal-agent contracts in dynamic settings in which both parties are allowed to freely borrow and lend without the knowledge of the other. ${ }^{5}$ But borrowing and lending contracts typically require some kind of outside enforcement, which in turn requires that they be observable by some aggregate institution.

\footnotetext{
${ }^{5}$ In Allen's model, as in ours, the agent receives income that is not observed by the principal. In such an environment, if the agent is allowed to freely borrow and lend without the knowledge of the principal, then only the Walrasian outcome is in fact incentive-feasible. In the setting that we study, with unobservable storage, there are many incentive-feasible outcomes with binding resource constraints; our main result is that the Walrasian allocation is in fact efficient relative to the other incentive-feasible outcomes.
} 
If we equate the aggregate institution undertaking the transfer system with a national government, then this is a serious weakness of Allen's and FHM's models, since this is the same institution which enforces domestic contracts. Private storage does not give rise to any need for outside enforcement.

Perhaps more importantly, another interpretation of private storage is that it represents the ability to save with some other agent which the aggregate institution cannot observe. Under this interpretation, there is some party that issues fully enforceable debt contracts. A well-known example of such agents are the Swiss banks who allow almost anyone to save with them and keep the magnitudes of these savings anonymous. It is an interesting question as to how these countries have overcome their incentive to repudiate this debt, but their having done so to a significant degree seems uncontestable. Hence from the perspective of a US institution, this ability to safely save abroad in a manner that is largely undetectable is equivalent to private storage. ${ }^{6}$

\section{The model world: feasibility and efficiency}

There is a continuum of identical agents who each live for three periods. There is a single consumption good in every period. In period $s$, individual preferences over consumption streams are representable by the utility function:

$$
E_{s} \sum_{t=s}^{3} \beta^{t-s} u\left(c_{t}\right) \text {, }
$$

where we assume that $u(\cdot)$ is continuous and concave, $u^{\prime}$ is positive and continuous, and that $\lim _{c \rightarrow 0} u^{\prime}(c)=\infty$. We also assume that $u$ displays nonincreasing absolute risk aversion; this

\footnotetext{
${ }^{6}$ Lim and Townsend (1996) document the importance of currency and grain as methods of saving and dissaving in south Indian villages. Townsend has argued that it seems unlikely that individual holdings of currency are observable to others in the village.
} 
implies that $u^{\prime}$ is strictly convex.

In each period $t$, an individual receives an unobservable stochastic endowment, $\theta_{t}$, which is drawn from a finite set $\Theta=\left\{\theta^{1}, \ldots, \theta^{J}\right\}$ of nonnegative reals. We assume that the probability of a draw is i.i.d. both across individuals and across time, and that the probability of an individual's receiving endowment $\theta^{j}$ is $\pi_{j}>0$; we also assume that $\theta^{i}<\theta^{i+1}$.

Goods are storable, with gross return equal to $R>0$. Individuals can store goods in a private storage unit that is unobservable to others, or in a public storage facility that is observable to all. We define an allocation to be a triple of individual consumption functions $\left(c_{1}, c_{2}, c_{3}\right)$, where $c_{t}$ maps $\Theta^{t}$ into the nonnegative reals, and a pair of individual storage functions $\left(s_{1}, s_{2}\right)$, where $s_{t}$ maps $\Theta^{t}$ into the nonnegative reals. An allocation is physically feasible if in each period, the per capita levels of consumption and storage are less than or equal to the sum of per capita endowments and the per capita proceeds from storage in the prior period.

An allocation mechanism is a description of how resources are to be distributed across agents. Specifically, an allocation mechanism consists of a message space $A$, a sequence of transfer functions $\left(\tau_{1}, \tau_{2}, \tau_{3}\right)$ such that $\tau_{t}$ maps $A^{t}$ into the reals, and a pair of storage levels $\left(S_{1}, S_{2}\right)$. In each period, after receiving his endowment, an agent sends a message (some selection from $A$ ) to a social planner; the transfer functions then determine the transfer of consumption to be made to the agent based on his current and past messages. (Of course, the transfer may be negative, in which case the agent gives resources to the planner.) The planner then publicly stores the amount of goods specified by the mechanism in the public storage facility. (Throughout the paper, $S_{t}$ represents public storage and $s_{t}$ represents private storage.) 
An allocation is incentive-feasible if it is implemented by some allocation mechanism; that is, the allocation is the Bayesian-Nash equilibrium outcome of agents' playing the game defined by the mechanism. It is simple to show that the Revelation Principle applies here. Hence, we can find the complete set of incentive-feasible allocations of resources by restricting attention to incentive-compatible mechanisms in which the message space $A$ is the same as the space $\Theta$ of endowment realizations, and in which it is weakly optimal for every individual to announce his true realization of $\theta$ given that he has always done so in the past.

Given this standard result, an allocation $\left(c_{1}, c_{2}, c_{3}, s_{1}, s_{2}\right)$ is incentive feasible if there exists an incentive-compatible mechanism $\left(\tau_{1}, \tau_{2}, \tau_{3}, S_{1}, S_{2}\right)$ such that

$$
\begin{aligned}
c_{1}\left(\theta^{i}\right) & =\theta^{i}+\tau_{1}\left(\theta^{i}\right)-s_{1}\left(\theta^{i}\right) \\
c_{2}\left(\theta^{i}, \theta^{j}\right) & =\theta^{j}+\tau_{2}\left(\theta^{i}, \theta^{j}\right)-s_{2}\left(\theta^{i}, \theta^{j}\right)+R s_{1}\left(\theta^{i}\right) \\
c_{3}\left(\theta^{i}, \theta^{j}, \theta^{k}\right) & =\theta^{k}+\tau_{3}\left(\theta^{i}, \theta^{j}, \theta^{k}\right)+R s_{2}\left(\theta^{i}, \theta^{j}\right)
\end{aligned}
$$

(2) $\left\{\sum_{i} \sum_{j} \sum_{k}\left[u\left(c_{1}\left(\theta^{i}\right)\right)+\beta u\left(c_{2}\left(\theta^{i}, \theta^{j}\right)\right) \pi_{j}+\beta^{2} u\left(c_{3}\left(\theta^{i}, \theta^{j}, \theta^{k}\right)\right)\right] \pi_{i} \pi_{j} \pi_{k}\right\}$

$$
\geq \max _{\substack{a_{1} \in \Theta, s_{1}^{\prime} \geq 0 \\
a_{2}\left(\theta^{j}\right) \in \Theta, s_{2}^{\prime}\left(\theta^{j}\right) \geq 0 \\
a_{3}\left(\theta^{j}, \theta^{k}\right) \in \Theta}}\left\{\begin{array}{c}
\sum_{i} \sum_{j} \sum_{k}\left[u\left(\theta^{i}+\tau_{1}\left(a_{1}\right)-s_{1}^{\prime}\right)+\beta u\left(\theta^{j}+\tau_{2}\left(a_{1}, a_{2}\left(\theta^{j}\right)\right)+R s_{1}^{\prime}-s_{2}^{\prime}\left(\theta^{j}\right)\right)\right. \\
\left.+\beta^{2} u\left(\theta^{k}+\tau_{3}\left(a_{1}, a_{2}\left(\theta^{j}\right), a_{3}\left(\theta^{j}, \theta^{k}\right)\right)+R s_{2}^{\prime}\left(\theta^{j}\right)\right)\right] \pi_{i} \pi_{j} \pi_{k}
\end{array}\right\}
$$

(3) $\quad \sum_{i} \pi_{i} \tau_{1}\left(\theta^{i}\right)+S_{1} \leq 0$

$$
\sum_{i} \sum_{j} \pi_{i} \pi_{j} \tau_{2}\left(\theta^{i}, \theta^{j}\right)+S_{2} \leq R S_{1}
$$

$$
\sum_{i} \sum_{j} \sum_{k} \pi_{i} \pi_{j} \pi_{k} \tau_{3}\left(\theta^{i}, \theta^{j}, \theta^{k}\right) \leq R S_{2}
$$

(6) $S_{1}, S_{2} \geq 0$ 


$$
c_{1}\left(\theta^{i}\right) \geq 0, c_{2}\left(\theta^{i}, \theta^{j}\right) \geq 0, c_{3}\left(\theta^{i}, \theta^{j}, \theta^{k}\right) \geq 0 .
$$

The last five conditions (3-7) guarantee that the allocation is physically feasible. The incentive constraint (2) ensures that the mechanism is in fact incentive-compatible by requiring that truth-telling is optimal. The ex-ante form of the incentive constraint may seem unfamiliar to some readers. Because period $t$ actions are allowed to be arbitrary functions of period $t$ information, this ex-ante incentive constraint is equivalent to writing down a sequence of ex-post incentive constraints.

The incentive constraint embeds the idea that to ensure that truth-telling is optimal, we have to trace out the full implications of deviating from truth-telling. It is important to note that the presence of the unobservable storage makes this task more difficult. Without unobservable storage, an individual finds truth-telling optimal even if he has defected from truth-telling in the past. In the environment that we are studying, failing to announce the truth may lead the individual to change his level of storage, and may thereby affect whether he finds truth-telling optimal in the following periods. In the language of Green (1987), it is not true that in this environment, temporary incentive-compatibility implies full incentivecompatibility.

Our goal in this paper is to characterize the set of symmetric efficient incentive-feasible allocation(s) (that is, efficient allocations in which all individuals receive the same ex-ante utility). Given the symmetry restriction, these allocations will be the solution to

$$
\begin{gathered}
\text { P1 : } \max _{\substack{\left(\tau_{1}, \tau_{2}, \tau_{3}\right) \\
\left(S_{1}, S_{2}\right)}} \sum_{i} u\left(c_{1}\left(\theta^{i}\right)\right) \pi_{i}+\beta \sum_{i} \sum_{j} u\left(c_{2}\left(\theta^{i}, \theta^{j}\right)\right) \pi_{i} \pi_{j}+ \\
\beta^{2} \sum_{i} \sum_{j} \sum_{k} u\left(c_{3}\left(\theta^{i}, \theta^{j}, \theta^{k}\right)\right) \pi_{i} \pi_{j} \pi_{k}
\end{gathered}
$$


subject to $(1)-(7)$.

It is not obvious how this problem can be made recursive. This is because there is not a set of publicly observable state variables upon which we can condition that is sufficient to determine individual outcomes from that point onwards. In the papers by Green (1987) and Atkeson and Lucas (1992), the continuation utility level promised under the mechanism, conditional upon the history of past reports, was sufficient. However, since different levels of private storage will lead individuals to value different sequences of transfers differently, this is not possible in our setting. In Khan and Ravikumar (1996) the level of storage is public information, and hence the state can be taken to be the level of storage $\times$ the utility promise. However, since here storage can be private information, this is not possible. This will force us to take a different approach to characterizing the symmetric efficient allocation.

\section{Characterizing efficient allocations}

In this section, we characterize the set of symmetric efficient allocations when either the gross return on storage is close to the inverse of the discount factor, or the lower bound of the support of the endowment distribution is close to zero. To do so, we first write down a simpler maximization problem, which we will label $P 2$, in which the constraint set is at least as large as the constraint set in $P 1$. We characterize the solution to this problem. Then, we show that the solution to this new optimization problem actually lies in $P 1$. We therefore conclude that the solution to the new optimization problem is in fact the solution to $P 1$.

In order to simplify $P 1$, note first that the incentive constraint (2) implies that reports in the last period will be chosen so as to maximize the level of the transfer, and hence it is 
incentive-infeasible for the transfer level in the third period to depend upon the realized shock. Therefore, without loss of generality, we can restrict the transfer in period three to simply be a function of the first two shocks and drop this incentive constraint entirely.

The next proposition also allows us to simplify much of the following discussion.

Proposition 1. Given any incentive-feasible allocation, there exists an incentive-compatible mechanism which implements the same consumption allocation and under which all individuals find it optimal not to store.

Proof. Assume that we have an incentive-compatible mechanism of the form $\left(\tau_{1}, \tau_{2}, \tau_{3}, S_{1}, S_{2}\right)$. Suppose that under this mechanism, individuals find it optimal to choose storage functions $\left\{s_{1}, s_{2}\right\}$. Then consider the following mechanism:

$$
\begin{aligned}
\tilde{\tau}_{1}\left(\theta^{i}\right) & =\tau_{1}\left(\theta^{i}\right)-s_{1}\left(\theta^{i}\right) \\
\tilde{\tau}_{2}\left(\theta^{i}, \theta^{j}\right) & =\tau_{2}\left(\theta^{i}, \theta^{j}\right)-s_{2}\left(\theta^{i}, \theta^{j}\right)+R s_{1}\left(\theta^{i}\right) \\
\tilde{\tau}_{3}\left(\theta^{i}, \theta^{j}\right) & =\tau_{3}\left(\theta^{i}, \theta^{j}\right)+R s_{2}\left(\theta^{i}, \theta^{j}\right) \\
\tilde{S}_{1} & =\sum_{i} \pi_{i} s_{1}\left(\theta^{i}\right)+S_{1} \\
\tilde{S}_{2} & =\sum_{i} \sum_{j} \pi_{i} \pi_{j} s_{2}\left(\theta^{i}, \theta^{j}\right)+S_{2} .
\end{aligned}
$$

We claim that this mechanism is also incentive-compatible and that it implements the same allocation. To see why, note that if an individual always tells the truth, he receives exactly the same consumption stream if he never stores as he did under the original mechanism when he saved optimally. Since he could have chosen to store more under the original mechanism, and did not, he will choose not to store in this new mechanism. 
Will any individual ever lie under the new mechanism? If he lies, then he receives a stream of consumption that he could have attained under the old mechanism by choosing the right stream of storage. Since he did not choose this stream of consumption, the utility associated with lying is no larger under the new mechanism than under the original mechanism. Hence, no individual finds it optimal to lie.

Consider the following alternative optimization problem.

$$
\begin{gathered}
\text { P2: } \max _{\substack{\left(\tau_{1}, \tau_{2}, \tau_{3}\right) \\
\left(S_{1}, S_{2}\right)}} \sum_{i} u\left(c_{1}\left(\theta^{i}\right)\right) \pi_{i}+\beta \sum_{i} \sum_{j} u\left(c_{2}\left(\theta^{i}, \theta^{j}\right)\right) \pi_{i} \pi_{j}+ \\
\beta^{2} \sum_{i} \sum_{j} \sum_{k} u\left(c_{3}\left(\theta^{i}, \theta^{j}, \theta^{k}\right)\right) \pi_{i} \pi_{j} \pi_{k}
\end{gathered}
$$

subject to

$$
\begin{aligned}
c_{1}\left(\theta^{i}\right) & =\theta^{i}+\tau_{1}\left(\theta^{i}\right) \\
c_{2}\left(\theta^{i}, \theta^{j}\right) & =\theta^{j}+\tau_{2}\left(\theta^{i}, \theta^{j}\right) \\
c_{3}\left(\theta^{i}, \theta^{j}, \theta^{k}\right) & =\theta^{k}+\tau_{3}\left(\theta^{i}, \theta^{j}\right)
\end{aligned}
$$

$$
\begin{aligned}
& \forall \theta^{i}: u\left(c_{1}\left(\theta^{i}\right)\right)+\beta \sum_{j} u\left(c_{2}\left(\theta^{i}, \theta^{j}\right)\right) \pi_{j}+\beta^{2} \sum_{j} \sum_{k} u\left(c_{3}\left(\theta^{i}, \theta^{j}, \theta^{k}\right)\right) \pi_{j} \pi_{k} \geq \\
& \max _{\substack{a_{1} \in\left\{\theta^{i}, \theta^{i-1}\right\} \\
s_{1}^{\prime}, s_{2}^{\prime}\left(\theta^{j}\right) \geq 0}} u\left(\theta^{i}+\tau_{1}\left(a_{1}\right)-s_{1}^{\prime}\right)+\beta \sum_{j} u\left(\theta^{j}+\tau_{2}\left(a_{1}, \theta^{j}\right)+R s_{1}^{\prime}-s_{2}^{\prime}\left(\theta^{j}\right)\right) \pi_{j} \\
& +\beta^{2} \sum_{j} \sum_{k} u\left(\theta^{k}+\tau_{3}\left(a_{1}, \theta^{j}\right)+R s_{2}^{\prime}\left(\theta^{j}\right)\right) \pi_{j} \pi_{k} \\
& \forall\left(\theta^{i}, \theta^{j}\right): u\left(c_{2}\left(\theta^{i}, \theta^{j}\right)\right)+\beta \sum_{k} u\left(c_{3}\left(\theta^{i}, \theta^{j}, \theta^{k}\right)\right) \pi_{k} \geq
\end{aligned}
$$




$$
\begin{aligned}
\max _{\substack{a_{2} \in\left\{\theta^{i}, \theta^{i-1}\right\} \\
s_{2}^{\prime} \geq 0}} u\left(\theta^{j}+\tau_{2}\left(\theta^{i}, a_{2}\right)-s_{2}^{\prime}\right)+ \\
\quad \beta \sum_{k} u\left(\theta^{k}+\tau_{3}\left(\theta^{i}, a_{2}\right)+R s_{2}^{\prime}\right) \pi_{k}
\end{aligned}
$$

$$
\begin{aligned}
& \sum_{i} \pi_{i} \tau_{1}\left(\theta^{i}\right)+S_{1} \leq 0 \\
& \sum_{i} \sum_{j} \pi_{i} \pi_{j} \tau_{2}\left(\theta^{i}, \theta^{j}\right)+S_{2} \leq R S_{1} \\
& \sum_{i} \sum_{j} \pi_{i} \pi_{j} \tau_{3}\left(\theta^{i}, \theta^{j}\right) \leq R S_{2}
\end{aligned}
$$

$$
S_{1}, S_{2} \geq 0
$$

$$
\tau_{1}\left(\theta^{i}\right) \geq-\theta^{i}, \tau_{2}\left(\theta^{i}, \theta^{j}\right) \geq-\theta^{j}, \tau_{3}\left(\theta^{i}, \theta^{j}\right) \geq-\theta^{1}
$$

To understand this problem, first recall that the endowment realizations are ordered from smallest to largest. Second, note that when $i=1, \theta^{i-1}$ is set equal to $\theta^{1}$. Third, note that we have taken advantage of proposition 1 . to restrict attention to solutions in which there is zero private savings. Finally, note that the third (last) period transfer is independent of the last period report to the planner, but cannot be more negative that the smallest possible endowment level.

The set of consumption allocations that satisfy the constraints of $P 2$ is larger than the set of mechanisms that satisfy the constraints of the original problem $P 1$. This is because the incentive constraints (11) and (12) in $P 2$ require that the mechanism be robust to a much smaller set of deviations. First, an individual can only tell one type of lie about himself: if he receives endowment realization $\theta^{i}$, he is allowed to claim that he received $\theta^{i-1}$, but he cannot claim that he received any other shock. (Agents who receive the lowest shock realization $\theta^{1}$ are not allowed to lie at all, but they are allowed to adjust their saving.) Second, after an 
individual lies in the current period, he is required to tell the truth in future periods, even though that behavior may be suboptimal. In the rest of this section, we provide necessary conditions for any solution to $P 2$ and show that these necessary conditions imply that any solution lies in the (smaller) constraint set of $P 1$.

We first prove that resource constraints must bind in any solution to $P 2$.

Proposition 2. In any solution to $P 2$, the aggregate resource constraints hold with equality.

Proof. Suppose the aggregate resource constraints don't hold with equality in period three. If this is so, in period three, give $\epsilon$ units of consumption to anyone who announces that he received the highest realization of $\theta$ in periods one and two. By the nature of the incentive constraints, no agent is allowed to claim that he got the highest shock if he did not, so the incentive constraints can't be violated. Also, giving more consumption to agents in period three will not lead them to save more. If the resource constraints don't hold with equality in periods 1 and 2, the planner should store $\epsilon$ units more consumption until period three and then give these extra resources to anyone who announces that he got the highest shock in periods one and two.

In what follows, the positive storage condition

$$
S_{t}>0 \text { for } t=1,2
$$

plays a central role. We prove that if all solutions to $P 2$ satisfy this condition, then the solutions to $P 2$ and $P 1$ are equivalent. We do so in three steps. First, any solution to $P 2$ that satisfies the positive storage condition must satisfy a consumption smoothing condition 
that says that individuals are marginally indifferent between storing and not storing, or

$$
\begin{aligned}
\text { for all } \theta^{i}, u^{\prime}\left(c_{1}\left(\theta^{i}\right)\right) & =\beta R \sum_{j} u^{\prime}\left(c_{2}\left(\theta^{i}, \theta^{j}\right)\right) \pi_{j} \\
(18) \text { for all }\left(\theta^{i}, \theta^{j}\right), u^{\prime}\left(c_{2}\left(\theta^{i}, \theta^{j}\right)\right) & =\beta R \sum_{k} u^{\prime}\left(c_{3}\left(\theta^{i}, \theta^{j}, \theta^{k}\right)\right) \pi_{k} .
\end{aligned}
$$

We then prove that any solution to $P 2$ that satisfies both of these conditions must also satisfy a net present value of transfers (NPV) condition that says that the sum of all transfers, discounted at the rate of return on storage, is the same across all individuals regardless of their shocks, and hence equal to zero, or

$$
\tau_{1}\left(\theta^{i}\right)+\tau_{2}\left(\theta^{i}, \theta^{j}\right) R^{-1}+\tau_{3}\left(\theta^{i}, \theta^{j}\right) R^{-2}=0 \text { for all } \theta^{i} \text { and } \theta^{j} .
$$

The third step is to show that any solution to $P 2$ which satisfies these three conditions will also satisfy the incentive condition (2) in $P 1$, and hence is a solution to that problem is as well.

The following proposition proves the first step in this chain of logic. The proof is simple: given an allocation in which per-capita storage is positive and some agent's zero storage constraint is binding, then the agent's utility can be improved by allowing him to "borrow" by lowering the amount being stored by the planner.

Proposition 3. Any solution to P2 that satisfies the positive storage condition (17) must also satisfy the consumption smoothing condition (18).

Proof. First, we show that in any solution to $P 2$ in which $S_{2}>0, u^{\prime}\left(c_{2}\right)=\beta R E_{2} u^{\prime}\left(c_{3}\right)$. Suppose this is not true for some $\left(\theta^{i}, \theta^{j}\right)$, and instead

$$
u^{\prime}\left(c_{2}\left(\theta^{i}, \theta^{j}\right)\right)>\beta R \sum_{k} u^{\prime}\left(c_{3}\left(\theta^{i}, \theta^{j}, \theta^{k}\right)\right) \pi_{k}
$$


Then, set $\tilde{S}_{2}=S_{2}-\epsilon \pi_{i} \pi_{j}$ units of consumption, $\tilde{\tau}_{2}\left(\theta^{i}, \theta^{j}\right)=\tau_{2}\left(\theta^{i}, \theta^{j}\right)+\epsilon$ units of consumption, and $\tilde{\tau}_{3}\left(\theta^{i}, \theta^{j}\right)=\tau_{3}\left(\theta^{i}, \theta^{j}\right)-\delta$, where

$$
u\left(c_{2}\left(\theta^{i}, \theta^{j}\right)+\epsilon\right)+\beta \sum_{k} u\left(c_{3}\left(\theta^{i}, \theta^{j}, \theta^{k}\right)-\delta\right) \pi_{k}=u\left(c_{2}\left(\theta^{i}, \theta^{j}\right)\right)+\beta \sum_{k} u\left(c_{3}\left(\theta^{i}, \theta^{j}, \theta^{k}\right)\right) \pi_{k},
$$

and

$$
u^{\prime}\left(c_{2}\left(\theta^{i}, \theta^{j}\right)+\epsilon\right)>\beta R \sum_{k} u^{\prime}\left(c_{3}\left(\theta^{i}, \theta^{j}, \theta^{k}\right)-\delta\right) \pi_{k}
$$

Clearly, since $u^{\prime}\left(c_{2}\left(\theta^{i}, \theta^{j}\right)\right)>\beta R \sum_{k} u^{\prime}\left(c_{3}\left(\theta^{i}, \theta^{j}, \theta^{k}\right)\right) \pi_{k}, \delta>\epsilon R$, and so this reshuffling is physically feasible and can be used to improve ex-ante utility. We still have to check the incentive constraints. Any individual who received the shock $\theta^{j+1}$ in period 2 would not announce $\theta^{j}$, because concavity implies that

$$
u\left(c_{2}\left(\theta^{i}, \theta^{j}\right)+\epsilon\right)-u\left(c_{2}\left(\theta^{i}, \theta^{j}\right)\right)>u\left(c_{2}\left(\theta^{i}, \theta^{j}\right)+\epsilon+\theta^{j+1}-\theta^{j}\right)-u\left(c_{2}\left(\theta^{i}, \theta^{j}\right)+\theta^{j+1}-\theta^{j}\right)
$$

and so

$$
\begin{aligned}
& u\left(c_{2}\left(\theta^{i}, \theta^{j}\right)+\theta^{j+1}-\theta^{j}+\epsilon\right)+\beta \sum_{k} u\left(c_{3}\left(\theta^{i}, \theta^{j}, \theta^{k}\right)-\delta\right) \pi_{k}< \\
& u\left(c_{2}\left(\theta^{i}, \theta^{j}\right)+\theta^{j+1}-\theta^{j}\right)+\beta \sum_{k} u\left(c_{3}\left(\theta^{i}, \theta^{j}, \theta^{k}\right)\right) \pi_{k} .
\end{aligned}
$$

Similarly, anyone who received the shock $\theta^{i+1}$ in period one would not announce $\theta^{i}$ under this new mechanism, because he gets the same utility as before from announcing $\theta^{i}$ and not storing after making this announcement, and they get less utility than before from announcing $\theta^{i}$ and choosing any positive level of storage (using the same concavity argument). Finally, any agent who announces $\theta^{j}$ will not choose to store now, because he is receiving more consumption in period 2. Hence, all individuals are marginally indifferent between storing or not in period 2 . 
If $S_{1}>0$, we can use the same logic as above that individuals are marginally indifferent between storing and not storing in period 1.

We have established our first step: if the positive storage condition is satisfied, then the consumption smoothing condition is satisfied. The next step is to describe how solutions to $P 2$ behave across states of nature if the positive storage and (by implication) consumption smoothing conditions are satisfied. The following proposition proves that any solution to $P 2$ that satisfies these conditions has the feature that every possible transfer sequence has the same net present value (or $N P V$ ), and so condition (19) holds as well.

Proposition 4. Any solution to P2 that satisfies the positive storage condition (17) and consumption smoothing condition (18) also satisfies the NPV condition (19).

\section{Proof. In Appendix.}

The proof is analytically tedious, but its logic is simple. We first note that any transfer scheme that gives an individual with a lower endowment shock a higher NPV sequence of transfers must lie outside the constraint set of $P 2$. Then we show that if the $N P V$ of the transfer to an individual with a higher endowment shock was also higher, then it is possible to construct a set of alternative transfers which yield the same ex-ante utility level and satisfy the incentive constraints, but require less resources.

Proposition 5. Any solution to P2 that satisfies the positive storage condition (17) is a solution to $P 1$.

Proof. To see this, we need only prove that any solution to $P 2$ that satisfies the positive storage condition must lie in the constraint set of $P 1$. Note first that any solution to $P 2$ 
in which storage is positive in periods 1 and 2 satisfies the $N P V$ property, so all transfer schemes deliver the same $N P V$ after any history of shocks. Also, if an agent tells the truth, his consumption is optimally smoothed. Hence, agents weakly prefer to tell the truth and not store, and so the constraints of $P 1$ are satisfied.

This proposition establishes the importance of the positive storage condition. To see this in a stark way, suppose the environment is specified in such a way that all solutions to $P 2$ satisfy the positive storage condition. Then, the proposition guarantees that the set of consumption allocations that solve $P 2$ is exactly the same as the set of consumption allocations that solve $P 1$. Thus, we know a lot about the symmetric efficient allocations (that is, the solutions to $P 1$ ) for any specification of the environment in which all solutions to $P 2$ satisfy the positive storage condition.

The next proposition provides two sets of conditions on $R, \beta$ and the support of $\theta$ that are sufficient to guarantee that the positive storage condition is satisfied for any solution to $P 2$. Essentially, we need either the return to storage to be sufficiently high, or the lowest possible realization of $\theta$ to be sufficiently low.

Proposition 6. All solutions to P2 satisfy the positive storage condition (17) if either of the following two conditions is satisfied:

$$
\begin{aligned}
& \text { i. } R \geq \beta^{-1} \\
& \text { ii. } \theta^{1}=0 .
\end{aligned}
$$

(Recall that $\theta^{1}$ is the lowest possible realization of $\theta$.)

Proof. In Appendix. 
The intuition behind case $(i)$ is that the incentive problem requires individuals to absorb some idiosyncratic risk into their consumptions. The presence of this idiosyncratic risk, combined with the convexity of marginal utility, means that the solutions to $P 2$ feature positive aggregate storage whenever $R \geq \beta^{-1}$. The intuition behind case (ii) is that because of the incentive problem, there is no insurance in period three. Hence, if $\theta^{1}=0$, then it is always optimal for society to store from periods two to three to guard against this bad outcome. We can then use backward induction to prove the theorem.

We can conclude that under either condition $(i)$ or condition (ii), any solution to $P 2$ is a solution to $P 1$, and hence any solution to $P 1$ must satisfy the positive storage condition, the consumption smoothing condition and the $N P V$ condition. It is worth noting that conditions (i) and (ii) can be relaxed. Since storage is strictly positive, the Theorem of the Maximum tells us that for small changes in the rate of return or small changes in $\theta^{1}$, per-capita storage must still be positive in any solution to $P 2$. Hence, the characterizations of the solutions to $P 1$ and $P 2$ are still valid for $R$ sufficiently close to $\beta^{-1}$ or for $\theta^{1}$ sufficiently low.

Finally, we want to show that if it is known that all solutions to $P 2$ satisfy the positive storage condition, then there is in fact a unique symmetric efficient allocation. We begin by showing that given this requirement, any allocation of consumption in a solution to $P 1$ actually solves the following optimization problem.

$$
\text { P3: } \max _{\substack{\tau_{1}, \tau_{2}, \tau_{3} \\\left(S_{1}, S_{2}\right)}} \sum_{i} \sum_{j} \sum_{k}\left[u\left(\theta^{i}+\tau_{1}\left(\theta^{i}\right)\right)+\beta u\left(\theta^{j}+\tau_{2}\left(\theta^{i}, \theta^{j}\right)\right)+\beta^{2} u\left(\theta^{k}+\tau_{3}\left(\theta^{i}, \theta^{j}\right)\right)\right] \pi_{i} \pi_{j} \pi_{k}
$$

subject to

$$
\tau_{1}\left(\theta^{i}\right)+\tau_{2}\left(\theta^{i}, \theta^{j}\right) R^{-1}+\tau_{3}\left(\theta^{i}, \theta^{j}\right) R^{-2}=0 \text { for all } \theta^{i}, \theta^{j}
$$




$$
\begin{aligned}
& \sum_{i} \tau_{1}\left(\theta^{i}\right) \pi_{i}+S_{1}=0 \\
& \sum_{i} \sum_{j} \tau_{2}\left(\theta^{i}, \theta^{j}\right)+S_{2}=R S_{1} \\
& \sum_{i} \sum_{j} \tau_{3}\left(\theta^{i}, \theta^{j}\right) \pi_{i} \pi_{j}=R S_{2} \\
& S_{1}, S_{2} \geq 0 \\
& \tau_{1}\left(\theta^{i}\right) \geq-\theta^{i}, \tau_{2}\left(\theta^{i}, \theta^{j}\right) \geq-\theta^{j}, \tau_{3}\left(\theta^{i}, \theta^{j}\right) \geq-\theta^{1}
\end{aligned}
$$

Note that the objective function in P3 is strictly concave, and the constraint set is convex. Hence, if we can find an element $\left(\tau_{1}, \tau_{2}, \tau_{3}, S_{1}, S_{2}\right)$ of the constraint set of $P 3$ such that $S_{t}>0$ for all $t$ and such that the first order conditions

$$
\begin{aligned}
u^{\prime}\left(\theta^{i}+\tau_{1}\left(\theta^{i}\right)\right) & =\beta R \sum_{j} \pi_{j} u^{\prime}\left(\theta^{j}+\tau_{2}\left(\theta^{i}, \theta^{j}\right)\right) \text { for all } \theta_{i} \\
u^{\prime}\left(\theta^{j}+\tau_{2}\left(\theta^{i}, \theta^{j}\right)\right) & =\beta R \sum_{k} \pi_{k} u^{\prime}\left(\theta^{k}+\tau_{3}\left(\theta^{i}, \theta^{j}\right)\right) \text { for all } \theta_{i}, \theta_{j}
\end{aligned}
$$

are satisfied, then $\left(\tau_{1}, \tau_{2}, \tau_{3}, S_{1}, S_{2}\right)$ is the unique solution to $P 3$.

Now, we know that the objective function in $P 2$ is continuous and the constraint set is compact. Hence, there is a solution to P2. Moreover, we know that any solution to P2 that satisfies the positive storage condition must also satisfy the consumption smoothing, and $N P V$ conditions. This means that any solution to $P 2$ lies in the constraint set of $P 3$ and satisfies the above first order conditions; any solution to $P 2$ that satisfies the positive storage condition is a solution to $P 3$. We can therefore deduce the following proposition.

Proposition 7. If all solutions to P2 are known to satisfy the positive storage condition, then the unique solution to P3 is the unique solution to P2; and furthermore all solutions to 
P1 have the same allocation of consumption as this unique solution to P2 and P3.

Note that there are always multiple solutions to $P 1$ because there are many efficient ways to divide per-capita storage between private and public storage. However, Proposition 7. guarantees that as long as all solutions to $P 2$ are known to satisfy the positive storage condition, then all solutions to $P 1$ feature the same allocation of consumption as in the unique solution to $P 3$.

\section{Supporting the efficient arrangement}

We now consider the equilibrium outcomes of a dynamic incomplete markets economy embedded in our physical and informational environment. In this trading arrangement, individuals competitively trade consumption and risk-free one period bonds that are available in zero net supply at each date. There is complete enforcement in this economy; individuals must pay off their debts in every state of the world, and they cannot end their lives in debt. We show if all solutions to $P 2$ are known to satisfy the positive storage condition (as is true,

for example, if $\beta R \geq 1$ or if $\theta^{1}=0$ ), then any symmetric efficient allocation is decentralizable in the sense that it is an equilibrium allocation for this incomplete markets economy. Also, we prove a partial converse that says that if $\beta R \geq 1$, or if $\theta^{1}=0$, then there is a unique equilibrium allocation and it is efficient.

The dynamic incomplete markets economy takes the following form. In each period, individuals trade consumption in exchange for risk-free bonds that promise one unit of consumption next period. The individuals treat interest rates (that is, the relative price between bonds and consumption in each period) as given. They derive wealth in a given period from public and private storage, from the interest payments on their bonds, and from their income. 
Individuals can use their wealth in three ways: they can store it publicly or privately, they can consume it, or they can use it to buy or sell bonds.

Hence, individuals choose consumption, bondholdings, private storage, and public storage $\left(c_{t}, b_{t}, s_{t}, \widetilde{s}_{t}\right)_{t=1}^{3}$, where each of the components of this vector is a function mapping $\Theta^{t}$ into the reals. Treating the interest rates $r_{1}$ and $r_{2}$ as fixed, the individuals make these choices so as to solve the following problem.

$$
\begin{aligned}
& \max E\left[u\left(c_{1}\right)+\beta u\left(c_{2}\right)+\beta^{2} u\left(c_{3}\right)\right] \\
& \text { subject to } \\
& t=1,2,3: c_{t}+b_{t}+s_{t}+\tilde{s}_{t} \leq \theta_{t}+b_{t-1}\left(1+r_{t}\right)+R s_{t-1}+R \tilde{s}_{t-1} \\
& b_{3} \geq 0 \\
& \tilde{s}_{t}, s_{t} \geq 0, t=1,2,3 \\
& b_{0}=0, s_{0}=0, \tilde{s}_{0}=0
\end{aligned}
$$

An equilibrium in this economy is a sequence of interest rates $\left(r_{1}, r_{2}\right)$, and a solution $\left(c_{t}, b_{t}, s_{t}, \tilde{s}_{t}\right)_{t=1}^{3}$ to the individual's decision problem such that for $t=1, . ., 3$ :

$$
\begin{aligned}
E\left\{b_{t}\right\} & =0 \\
E\left\{c_{t}\right\}+E\left\{s_{t}\right\}+E\left\{\tilde{s}_{t}\right\} & =R E\left\{s_{t-1}\right\}+R E\left\{\tilde{s}_{t-1}\right\}
\end{aligned}
$$

where $E$ represents the unconditional expectation of the random variable. The first equation requires that per-capita bondholdings equal zero, and the second equation requires that percapita demand and per-capita supply are equated in the goods market. 
Given this definition of equilibrium, we can prove that any symmetric efficient allocation is decentralizable.

Proposition 8. Suppose it is known that all solutions to P2 satisfy the positive storage condition. Then, the unique symmetric efficient allocation of consumption is a dynamic incomplete markets equilibrium allocation of consumption.

Proof. We have already established that if all solutions to P2 are known to satisfy the positive storage condition, then the unique symmetric efficient allocation is a solution to $P 3$. So, consider any solution $\left(\tau_{1}, \tau_{2}, \tau_{3}, S_{1}, S_{2}\right)$ to $P 3$. Define

$$
\begin{aligned}
& b_{1}=-S_{1}-\tau_{1} \\
& b_{2}=-\tau_{2}-S_{2}+R S_{1}+R b_{1} \\
& b_{3}=-\tau_{3}+R S_{2}+R b_{2} \\
& s_{1}=0 ; \quad \tilde{s}_{1}=S_{1} \\
& s_{2}=0 ; \quad \tilde{s}_{2}=S_{2} \\
& s_{3}=0 ; \quad \tilde{s}_{3}=0 .
\end{aligned}
$$

We claim that this is an equilibrium allocation in which the equilibrium interest rates are both equal to $R-1$. We know that in the solution to $P 3$, the individual's first order conditions are satisfied because consumption is smoothed. Also, the aggregate resource constraints are satisfied because the per-capita bond holdings are zero from the resource constraints in P3. We need only show that the solution to $P 3$ satisfies the individual budget constraints in 
the asset market. The period $t$ flow constraint follows from the reasoning

$$
\begin{aligned}
\theta_{t}-S_{t}+R S_{t-1}-b_{t}+R b_{t-1} & =\theta_{t}-S_{t}+R S_{t-1}+\tau_{t}+S_{t}-R S_{t-1} \\
& =\theta_{t}+\tau_{t} \\
& =c_{t} .
\end{aligned}
$$

Also, the constraint that $b_{3} \geq 0$ is a consequence of

$$
\begin{aligned}
b_{3} & =-\tau_{3}+R S_{2}+R b_{2} \\
& =-\tau_{3}-R \tau_{2}+R^{2} S_{1}+R^{2} b_{1} \\
& =-\tau_{3}-R \tau_{2}-R^{2} \tau_{1} \\
& =0 .
\end{aligned}
$$

It follows that the allocation of consumption in the solution to $P 3$ (that is, to $P 1$ ) is an equilibrium allocation of consumption.

Finally, we can show that if the gross rate of return on storage is at least $\beta^{-1}$, or zero is the lower support of the endowment distribution, then the reverse holds as well.

Proposition 9. If $R \geq \beta^{-1}$, or if $\theta^{1}=0$, then any dynamic incomplete markets equilibrium allocation of consumption is the unique symmetric efficient allocation of consumption.

Proof. We first prove that if $R \geq \beta^{-1}$ or $\theta^{1}=0$, then all agents must smooth consumption over time in any equilibrium allocation. Consider any equilibrium allocation in which percapita storage is positive at the end of period $t$. It is immediate in such an equilibrium that 
$r_{t}$ equals $R-1$ and that consumption is smoothed over time:

$$
\begin{aligned}
& u^{\prime}\left(c_{1}\right)=R \beta E_{1} u^{\prime}\left(c_{2}\right) \\
& u^{\prime}\left(c_{2}\right)=R \beta E_{2} u^{\prime}\left(c_{3}\right) .
\end{aligned}
$$

where $E_{t}$ represents the expectation conditional on $\left(\theta_{1}, \ldots, \theta_{t}\right)$. Hence, if per-capita storage is positive, then all agents smooth consumption.

Now suppose that $R \geq \beta^{-1}$. Suppose there is an equilibrium in which consumption is not smoothed from periods $t$ to $(t+1)$ for all individuals. Then for some history of shocks through period $t$, and when we use the convexity of $u^{\prime}$,

$$
u^{\prime}\left(c_{t}\right)>R \beta E_{t} u^{\prime}\left(c_{t+1}\right) \geq u^{\prime}\left(E_{t} c_{t+1}\right)
$$

It follows from the concavity of $u$ that

$$
E\left\{c_{t}\right\}<E\left\{c_{t+1}\right\}
$$

and so per-capita storage is positive from period $t$ to period $(t+1)$. But from the argument in the previous paragraph, we know that this contradicts the hypothesis that consumption is not smoothed for everyone.

Now suppose that $\theta^{1}=0$ and $R>0$. Suppose there is an equilibrium in which consumption is not smoothed between periods $t$ and $(t+1)$ for some individual. In this case, $b_{t} \geq 0$. Then for all $\theta_{t}>\theta^{1}$,

$$
u^{\prime}\left(\theta_{t}+\left(1+r_{t}\right) b_{t-1}+R\left(s_{t}+\widetilde{s}_{t}\right)\right)<R \beta E_{t} u^{\prime}\left(\theta_{t+1}\right) \leq R \beta E_{t} u^{\prime}\left(c_{t+1}\right),
$$

and so any individual with a positive income in period $t$ wants to store. Hence, zero storage cannot occur in equilibrium in this case as well. 
Next, construct a transfer/storage scheme by defining $\tau_{t} \equiv \theta_{t}-c_{t}$ and defining $S_{t}$ to be per-capita storage (both public and private) in equilibrium. This must be an element of $P 3$ 's constraint set because the $N P V$ of the difference between income and consumption must be zero in the equilibrium. Moreover, because consumption smoothing is satisfied, this must be a solution to $P 3$ (and also to $P 1$ ). Thus, any equilibrium allocation of consumption is efficient.

The conditions in proposition 9. are sufficient but not necessary. Since total storage is strictly positive under the conditions of the proposition, we can once again make us of the Theorem of the Maximum to argue that for returns on storage close to $\beta^{-1}$ or endowment distributions where the lower support is close to zero, our results will go through.

\section{Concluding Comments}

In this paper, we consider a simple environment in which individuals receive income shocks that are unobservable to others and can privately store resources. We have established several important characteristics of efficient arrangements for this environment if the rate of return on savings is not too much less than the rate of time preference, or, alternatively, if the worst possible outcome is sufficiently dire. The presence of the unobservable storage forces all efficient arrangements to smooth consumption over time. This severely reduces the ability of the society to share risk, as can be seen from the fact that after any history of shocks, there is no transfer of wealth among agents with different endowment realizations. We also show in this case that the symmetric efficient allocation is decentralizable through a dynamic incomplete markets trading arrangement in which individuals competitively trade risk-free bonds among themselves and face infinite costs of dying in debt. Thus, apparently ad hoc 
restrictions on the securities available for trade are consistent with the implementation of the efficient allocation in this setting.

We prove our results in a three period environment since this allows us to reduce the notational complexity, and still have it be easy to see how to generalize our results to any finite horizon. While many of the papers in this literature have used infinite horizon models, this choice appears to be one of analytic convenience. This is in particular true of the result that efficient allocations cannot be decentralized using competitive trade in various securities. Thomas and Worrall (1990) formally show that in their model that the infinite horizon efficient allocation is the limit of a sequence of efficient allocations of finite horizon models. Extending our results to an infinite horizon is an interesting topic for future research. Since our current results depend on a backward induction argument, it is not obvious how this can be done. 


\section{Appendix}

\section{Appendix}

\section{A1. Proof of proposition 4.:}

Consider some $\theta^{i}$ and some $\theta^{j}$. Suppose first that in a solution to $P 2, \tau_{2}\left(\theta^{i}, \theta^{j}\right)+$ $\tau_{3}\left(\theta^{i}, \theta^{j}\right) / R<\tau_{2}\left(\theta^{i}, \theta^{j-1}\right)+\tau_{3}\left(\theta^{i}, \theta^{j-1}\right) / R$. Condition (18) implies that $\theta^{j-1}$ agents are marginally indifferent between storing and not storing when they tell the truth. This means that since $\theta^{j}$ agents receive more endowment in period 2, they are not storage-constrained if they announce $\theta^{j-1}$. But then $\theta^{j}$ individuals should announce $\theta^{j-1}$ to get the higher $N P V$ transfer scheme.

Now suppose that $\tau_{2}\left(\theta^{i}, \theta^{j}\right)+\tau_{3}\left(\theta^{i}, \theta^{j}\right) / R>\tau_{2}\left(\theta^{i}, \theta^{j-1}\right)+\tau_{3}\left(\theta^{i}, \theta^{j-1}\right) / R$. Since $\theta^{j}$ agents are not storage-constrained by either transfer scheme, they strictly prefer telling the truth to lying:

$$
\begin{aligned}
& u\left(\theta^{j}+\tau_{2}\left(\theta^{i}, \theta^{j}\right)\right)+\beta \sum_{k} u\left(\theta^{k}+\tau_{3}\left(\theta^{i}, \theta^{j}\right)\right) \pi_{k}> \\
& \max _{s \geq 0} u\left(\theta^{j}+\tau_{2}\left(\theta^{i}, \theta^{j-1}\right)-s\right)+\beta \sum_{k} u\left(\theta^{k}+\tau_{3}\left(\theta^{i}, \theta^{j-1}\right)+R s\right) \pi_{k}
\end{aligned}
$$

We claim that we can construct a new mechanism that satisfies the constraints of $P 2$ and increases the objective. Define a new mechanism $\left(\tilde{\tau}_{2}, \tilde{\tau}_{3}\right)$ which is identical to the previous one, except

$$
\begin{aligned}
\tilde{\tau}_{2}\left(\theta^{i}, \theta^{j}\right) & =\tau_{2}\left(\theta^{i}, \theta^{j}\right)-\epsilon \\
\tilde{\tau}_{2}\left(\theta^{i}, \theta^{j-1}\right) & =\tau_{2}\left(\theta^{i}, \theta^{j-1}\right)+\psi(\epsilon) \pi_{j} / \pi_{j-1}
\end{aligned}
$$




$$
\begin{aligned}
\tilde{\tau}_{3}\left(\theta^{i}, \theta^{j}\right) & =\tau_{2}\left(\theta^{i}, \theta^{j-1}\right)-R \delta(\epsilon) \\
\tilde{\tau}_{3}\left(\theta^{i}, \theta^{j-1}\right) & =\tau_{3}\left(\theta^{i}, \theta^{j-1}\right)+R \gamma(\epsilon) \pi_{j} / \pi_{j-1}
\end{aligned}
$$

where $(\epsilon, \psi(\epsilon), \gamma(\epsilon), \delta(\epsilon))$ satisfy the following requirements

$$
\begin{aligned}
& \sum_{j}\left[u\left(\theta^{j}+\tilde{\tau}_{2}\left(\theta^{i}, \theta^{j}\right)\right)+\beta \sum_{k} u\left(\theta^{k}+\tilde{\tau}_{3}\left(\theta^{i}, \theta^{j}\right)\right) \pi_{k}\right] \pi_{j} \\
= & \sum_{j}\left[u\left(\theta^{j}+\tau_{2}\left(\theta^{i}, \theta^{j}\right)\right)+\beta \sum_{k} u\left(\theta^{k}+\tau_{3}\left(\theta^{i}, \theta^{j}\right)\right) \pi_{k}\right] \pi_{j}
\end{aligned}
$$

$$
\begin{aligned}
u^{\prime}\left(\theta^{j}+\tilde{\tau}_{2}\left(\theta^{i}, \theta^{j}\right)\right) & =\beta R \sum_{k} u^{\prime}\left(\theta^{k}+\tilde{\tau}_{3}\left(\theta^{i}, \theta^{j}\right)\right) \pi_{k} \\
u^{\prime}\left(\theta^{j-1}+\tilde{\tau}_{2}\left(\theta^{i}, \theta^{j-1}\right)\right) & =\beta R \sum_{k} u^{\prime}\left(\theta^{k}+\tilde{\tau}_{3}\left(\theta^{i}, \theta^{j-1}\right)\right) \pi_{k}
\end{aligned}
$$

Hence, the new mechanism takes from the person with the higher endowment shock in period two and gives to the person with the lower endowment shock in period two, while leaving unchanged the ex-ante utility level conditional on the period one endowment shock. The changes are smoothed so that the agents are marginally indifferent between storing and not storing.

We want to show that this new mechanism gives rise to extra resources (that can be given to the individuals in period three who claimed the high shock in the previous two periods). To do this, we totally differentiate the ex-ante utility of someone who announced 
$\theta^{i}$ in period 1 with respect to $\epsilon$ around $\epsilon=0$ :

$$
\begin{aligned}
0=- & u^{\prime}\left(\theta^{j}+\tau_{2}\left(\theta^{i}, \theta^{j}\right)\right) \pi_{j}-\beta R \sum_{k} \pi_{k} u^{\prime}\left(\theta^{k}+\tau_{3}\left(\theta^{i}, \theta^{j}\right)\right) \pi_{j} \delta^{\prime}(0) \\
& \quad+u^{\prime}\left(\theta^{j-1}+\tau_{2}\left(\theta^{i}, \theta^{j-1}\right)\right) \pi_{j} \psi^{\prime}(0)+\beta R \sum_{k} \pi_{k} u^{\prime}\left(\theta^{k}+\tau_{3}\left(\theta^{i}, \theta^{j-1}\right)\right) \pi_{j} \gamma^{\prime}(0) \\
=- & -u^{\prime}\left(\theta^{j}+\tau_{2}\left(\theta^{i}, \theta^{j}\right)\right) \pi_{j}\left(1+\delta^{\prime}(0)\right)+u^{\prime}\left(\theta^{j-1}+\tau_{2}\left(\theta^{i}, \theta^{j-1}\right)\right) \pi_{j}\left(\psi^{\prime}(0)+\gamma^{\prime}(0)\right) .
\end{aligned}
$$

Agent $j$ receives more consumption in period 2 than agent $(j-1)$, because both are smoothing and agent $j$ gets a higher $N P V$ transfer scheme. This implies that agent $j$ 's marginal utility is lower than $(j-1)$ 's, and so $\left(1+\delta^{\prime}(0) / R\right)>\left(\psi^{\prime}(0)+\gamma^{\prime}(0) / R\right)$. Hence, at least for small $\epsilon$, the new mechanism frees up resources (note that this conclusion exploits the result from the above Proposition that $S_{2}>0$ ).

We now want to show that the new mechanism satisfies the truth-telling and zerostorage constraints of $P 2$. We know that neither $\theta^{j}$ agents or $\theta^{j-1}$ agents will want to store more. As long as $\epsilon$ is sufficiently small, $\theta^{j}$ agents will not want to switch to $\theta^{j-1}$; also, $\theta^{j+1}$ agents will not switch to $\theta^{j}$ because the new mechanism makes announcing $\theta^{j}$ less attractive than before.

This serves to verify that the new mechanism satisfies the truth-telling and zero-storage constraints in period two. Now we have to check period one. First, will $\theta^{i}$ agents want to store more? To answer this, define

$$
\begin{aligned}
& V_{2}(W) \equiv \max _{c, s^{\prime}} u(c)+\beta \sum_{k} u\left(\theta^{k}+s^{\prime} R\right) \pi_{k} \\
& \text { s.t. } \quad c+s^{\prime} \leq W .
\end{aligned}
$$

Given any mechanism that smooths consumption from period two to period three, type $i$ 
agents will not want to store if

$$
u^{\prime}\left(\theta^{i}+\tau_{1}\left(\theta^{i}\right)\right) \geq R \beta \sum_{j} V_{2}^{\prime}\left(W_{j}\right) \pi_{j}
$$

where $W_{j} \equiv \theta^{j}+\tau_{2}\left(\theta^{i}, \theta^{j}\right)+\tau_{3}\left(\theta^{i}, \theta^{j}\right) / R$. We know that under the old mechanism, the above marginal condition was in fact an equality. The new mechanism reduces $W_{j}$ by $\epsilon+\delta(\epsilon) / R$ and increases $W_{j-1}$ by $[\psi(\epsilon)+\gamma(\epsilon) / R] \pi_{j} / \pi_{j-1}$. Totally differentiating $\sum_{j} V_{2}^{\prime}\left(W_{j}\right) \pi_{j}$ with respect to $\epsilon$ around $\epsilon=0$, we get

$$
\begin{aligned}
& -V_{2}^{\prime \prime}\left(W_{j}\right) \pi_{j}\left(1+\delta^{\prime}(0) / R\right)+V_{2}^{\prime \prime}\left(W_{j-1}\right)\left(\psi^{\prime}(0)+\gamma^{\prime}(0) / R\right) \pi_{j} \\
= & {\left[-V_{2}^{\prime \prime}\left(W_{j}\right) \pi_{j} / V_{2}^{\prime}\left(W_{j}\right)+V_{2}^{\prime \prime}\left(W_{j-1}\right) \pi_{j} / V_{2}^{\prime}\left(W_{j-1}\right] * k,\right.}
\end{aligned}
$$

where the equality with the second expression came from noting that (A2) implies that we can define

$$
k \equiv u^{\prime}\left(\theta^{j}+\tau_{2}\left(\theta^{i}, \theta^{j}\right)\right) \pi_{j}\left(1+\delta^{\prime}(0) / R\right)=u^{\prime}\left(\theta^{j-1}+\tau_{2}\left(\theta^{i}, \theta^{j-1}\right)\right) \pi_{j}\left(\psi^{\prime}(0)+\gamma^{\prime}(0) / R\right),
$$

where $k$ is a positive number. We know from Neave (1971) that if $u$ exhibits nonincreasing absolute risk aversion, so does $V_{2}$. It follows that since $W_{j}>W_{j-1}$, the total derivative is negative; for small $\epsilon$, the new mechanism makes $\theta^{i}$ agents less willing to store in period 1 .

In period one, do $\theta^{i+1}$ agents want to lie under the new mechanism? If they do so, then they get the same utility from lying and not storing as they would under the old mechanism. If they lie and store, then by using a similar argument as in the above paragraph, we can conclude that the new mechanism gives them less utility than the old mechanism.

This proves that in any solution to $P 2, \tau_{2}\left(\theta^{i}, \theta^{j}\right)+\tau_{3}\left(\theta^{i}, \theta^{j}\right) / R$ is independent of $\theta^{j}$. Now suppose that in some solution to $P 2, \tau_{1}\left(\theta^{i}\right)+\tau_{2}\left(\theta^{i}, \theta^{j}\right) / R+\tau_{3}\left(\theta^{i}, \theta^{j}\right) / R^{2}>\tau_{1}\left(\theta^{i-1}\right)+$ 
$\tau_{2}\left(\theta^{i-1}, \theta^{j}\right) / R+\tau_{3}\left(\theta^{i-1}, \theta^{j}\right) / R^{2}$. Lower $\tau_{1}\left(\theta^{i}\right)$ by $\epsilon$ units of consumption and raise $\tau_{3}\left(\theta^{i-1}, \theta^{j}\right)$ by $R^{2} \epsilon \pi_{i} / \pi_{i-1}$ for all $j$. Since $\theta^{i}+\tau_{1}\left(\theta^{i}\right)>\theta^{i-1}+\tau_{1}\left(\theta^{i-1}\right)$, this increases ex-ante utility. If $\epsilon$ is small enough, $\theta^{i}$ agents will not want to lie. Also, the change is designed so that the agents do not want to store.

It follows that the $N P V$ of agent $\theta^{i}$ s transfer scheme must be no larger than that of $\theta^{i-1}$. However, because any solution to $P 2$ smooths consumption, we know that the $N P V$ of agent $\theta^{i}$ 's transfer scheme can't be smaller than that of $\theta^{i-1}$. Physical feasibility then dictates that $\tau_{1}\left(\theta^{i}\right)+\tau_{2}\left(\theta^{i}, \theta^{j}\right) / R+\tau_{3}\left(\theta^{i}, \theta^{j}\right) / R^{2}=0$ for all $\theta^{i}$ and $\theta^{j}$. The theorem follows.

\section{A2. Proof of proposition 6.:}

We first prove $(i)$. We know individuals must find it optimal not to store any consumption from period two to period three; thus,

$$
0 \in \arg \max _{s_{2} \geq 0} u\left(c_{2}-s_{2}\right)+\beta E_{2} u\left(c_{3}+R s_{2}\right) .
$$

This implies that

$$
u^{\prime}\left(c_{2}\right) \geq \beta R E_{2} u^{\prime}\left(c_{3}\right)
$$

From the strict convexity of $u^{\prime}($.$) , it follows that E_{2} u^{\prime}\left(c_{3}\right) \geq u^{\prime}\left(E_{2} c_{3}\right)$, where this inequality is strict if $c_{3}$ depends on period 3 endowment shocks (for any realization of endowment shocks in period 2 or before). We know that for all $\left(\theta^{i}, \theta^{j}\right), c_{3}$ depends on $\theta_{3}$, because $\tau_{3}$ is independent of $\theta_{3}$. This implies that

$$
\text { for all }\left(\theta^{i}, \theta^{j}\right), u^{\prime}\left(c_{2}\left(\theta^{i}, \theta^{j}\right)\right) \geq \beta R \sum_{k} \pi_{k} u^{\prime}\left(c_{3}\left(\theta^{i}, \theta^{j}, \theta^{k}\right)\right)>u^{\prime}\left(\sum_{k} \pi_{k} c_{3}\left(\theta^{i}, \theta^{j}, \theta^{k}\right)\right)
$$

and in turn, it follows that

$$
\sum_{i} \sum_{j} \sum_{k}\left[c_{2}\left(\theta^{i}, \theta^{j}\right)-c_{3}\left(\theta^{i}, \theta^{j}, \theta^{k}\right)\right] \pi_{i} \pi_{j} \pi_{k}<0
$$


which implies that $S_{2}>0$, since we know from Proposition 2 that the resource constraints hold with equality.

We now prove that $S_{1}>0$. We proceed as we did in regard to $S_{2}$. Suppose $c_{2}\left(\theta^{i}, \theta^{j}\right)$ is independent of $\theta^{j}$. From Proposition 3., we know that $u^{\prime}\left(c_{2}\left(\theta^{i}, \theta^{j}\right)\right)=\beta R \sum_{k} \pi_{k} u^{\prime}\left(c_{3}\left(\theta^{i}, \theta^{j}, \theta^{k}\right)\right)$, and so $\sum_{k} \pi_{k} u^{\prime}\left(c_{3}\left(\theta^{i}, \theta^{j}, \theta^{k}\right)\right)$ is independent of $\theta^{j}$. It follows that $\tau_{3}\left(\theta^{i}, \theta^{j}\right)$ cannot depend on $\theta^{j}$. But then the incentive constraints can only be satisfied if $\tau_{2}\left(\theta^{i}, \theta^{j}\right)$ is independent of $\theta^{j}$, which contradicts the hypothesis that $c_{2}\left(\theta^{i}, \theta^{j}\right)$ is independent of $\theta^{j}$. We can then exploit the convexity of $u^{\prime}$ as above to conclude that $S_{1}>0$. This completes the proof of $(i)$.

We next prove $(i i)$. We know from condition (16) that $\tau_{3}\left(\theta_{i}, \theta_{j}\right) \geq 0$ for all $i, j$ (since the individual could receive an endowment of 0 in the last period). Next, we know from proposition 2. that the resource constraint binds in every period. Hence in each period, either savings is positive or consumption is positive for some type. If second period consumption was zero for all types, then $S_{2}>0$, but if for some $i, j, c_{2}\left(\theta^{i}, \theta^{j}\right)>0$, then for these agents

$$
u^{\prime}\left(c_{2}\left(\theta_{i}, \theta_{j}\right)\right) \geq \beta R \sum_{k} \pi_{k} u^{\prime}\left(\theta^{k}+\tau_{3}\left(\theta^{i}, \theta^{j}\right)\right)
$$

This along with the assumption that $u^{\prime}(0)=\infty$ implies that $\tau_{3}\left(\theta_{i}, \theta_{j}\right)>0$. Physical feasibility would then tell us that $S_{2}>0$.

Given that $S_{2}$ is positive, we know from Proposition 3. that (A3) holds as an equality. We can then proceed as in the proof of proposition 4. to establish the $N P V$ property with respect to the last two periods:

$$
\tau_{2}\left(\theta^{i}, \theta^{j}\right)+\tau_{3}\left(\theta^{i}, \theta^{j}\right) / R \text { is the same for all } j \text {. }
$$


Physical feasibility dictates that $\tau_{2}\left(\theta^{i}, \theta^{1}\right) \geq 0$. Hence, from our $N P V$ property for the last two periods, and given that $\tau_{3}\left(\theta_{i}, \theta_{j}\right) \geq 0$, it follows that:

$$
\tau_{2}\left(\theta^{i}, \theta^{j}\right)+\tau_{3}\left(\theta^{i}, \theta^{j}\right) / R \geq 0 \text { for all } i
$$

and furthermore that this inequality is strict for any $(i, j)$ such that $\tau_{2}\left(\theta^{i}, \theta^{j}\right)>0$.

For any agent $\theta^{i}$ such that $c_{1}\left(\theta^{i}\right)>0$, either $\tau_{2}\left(\theta^{i}, \theta^{1}\right)>0$ or he will store for any $R$. Hence the $N P V$ of the transfer scheme going to $\left(\theta^{i}, \theta^{1}\right)$ from the second period onwards (which is given by A4) is positive. Thus, $S_{1}>0$ must hold.

\section{References}

[1] Aiyagari, Rao. "Uninsured Idiosyncratic Risk and Aggregate Savings," Quarterly Journal of Economics 109(3), (August 1994), pages 659-684.

[2] Aiyagari, Rao and Fernando Alvarez. "Stationary Efficient Distributions with Private Information and Monitoring: A Tale of Kings and Slaves," manuscript, Federal Reserve Bank of Minneapolis (1995).

[3] Abreu, Dilip, David Pearce and Ennio Stacchetti. "Toward a Theory of Discounted Repeated Games with Imperfect Monitoring", Econometrica, 58, (1990), pages 10411064.

[4] Allen, Franklin. "Repeated Principal-Agent Relationships with Lending and Borrowing", Economic Letters 17, (1985), pages 27-31.

[5] Atkeson, Andrew. "International Lending with Moral Hazard and Risk of Repudiation," Econometrica 59(4), (1991), pages 1069-1089. 
[6] Atkeson, Andrew and Robert Lucas, Jr.. "On Efficient Distribution with Private Information," The Review of Economic Studies; 59(3), (July 1992), pages 427-454.

[7] Atkeson, Andrew and Robert Lucas, Jr.. "Efficiency and Equality in a Simple Model of Efficient Unemployment Insurance", Journal of Economic Theory, 66(1), (June 1995), pages $64-88$.

[8] Backus, David, Patrick Kehoe and Finn Kydland. "International Real Business Cycles", Journal of Political Economy 100, (1992), pages 745-775.

[9] Bewley, Truman. "Thoughts on Volatility Tests of the Intertemporal Asset Pricing Model," in Robert Becker, Michele Boldrin, Ronald Jones, and William Thomson, eds., General Equilibrium, Growth, and Trade II: The Legacy of Lionel Mackenzie (San Diego: Academic Press, Inc., 1993).

[10] Carroll, Christopher. "The Buffer Stock Theory of Savings: Some Macroeconomic Evidence," Brookings Papers on Economic Activity 0(2), (1992), pages 61-135.

[11] Cochrane, John. "A Simple Test of Consumption Insurance," Journal of Political Economy 99 (October 1991), pages 957-76.

[12] Cole, Harold and Patrick Kehoe. "The Role of Institutions in Reputation Models of Sovereign Debt," Journal of Monetary Economics 35, (1995), pages 45-64.

[13] Deaton, Angus. Understanding Consumption, (Oxford, UK: Clarendon Press, 1992).

[14] Deaton, Angus and Christina Paxson. "Intertemporal Choice and Inequality," Journal of Political Economy 102(3), (June 1994), pages 437-467. 
[15] Fudenberg, D., Holmstrom, B., and P. Milgrom. "Short-Term Contracts and Long-Term Agency Relationships," Journal of Economic Theory 51, (1990), pages 1-31.

[16] Geanakoplos, John. "An Introduction to General Equilibrium with Incomplete Asset Markets," Journal of Mathematical Economics 19(1/2), (1990), pages 1-38.

[17] Green, Edward. "Lending and the Smoothing of Uninsurable Income", in Edward Prescott and Neil Wallace, eds., Contractual Arrangements for Intertemporal Trade, (Minneapolis: University of Minnesota Press, 1987).

[18] Green, Edward, and Soo-Nam Oh. "Contracts, Constraints, and Consumption," Review of Economic Studies 58, (1991), 883-900.

[19] Hayashi, Fumio, Altonji, Joseph, and Laurence Kotlikoff. "Risk-Sharing between and within Families," Econometrica 64(2), (1996), pages 261-294.

[20] Huggett, Mark. "The Risk-Free Rate in Heterogeneous-Agent Incomplete-Insurance Economies," Journal of Economic Dynamics and Control 17, (1993), 953-969.

[21] Kehoe, Timothy and David Levine. "Debt Constrained Asset Markets," Review of Economic Studies, 60 (1993), pages 865-888.

[22] Khan, Aubik and B. Ravikumar (1996), "Growth and Distribution with Private Information," manuscript, University of Virginia.

[23] Marcet, Albert and Ramon Marimon. "Communication, Commitment and Growth," Journal of Economic Theory, 58(2), (December 1992), pages 219-49. 
[24] Neave, Edwin H. "Multiperiod Consumption-Investment Decisions and Risk Preference," Journal of Economic Theory 3, (1971), pages 40-53.

[25] Phelan, Christopher and Robert Townsend. "Computing Multi-Period, Information Constrained Optima," Review of Economic Studies 58, (1991), pages 853-881.

[26] Phelan, Christopher. "Repeated Moral Hazard and One-Sided Commitment," Journal of Economic Theory 66(2), (August 1995), pages 488-506.

[27] Spear, Stephen and Sanjay Srivastava. "On Repeated Moral Hazard with Discounting," Review of Economic Studies 54, (1987), pages 599-617.

[28] Schmeidler, David and Karl Vind. "Fair Net Trades," Econometrica 40(4), (July 1972), pages $637-42$.

[29] Taub, Bart. "Dynamic Mechanisms on a Continuum," manuscript, University of Illinois, (1990).

[30] Thomas, Jonathan and Thomas Worrall. "Income Fluctuations and Asymmetric Informations: An Example of a Repeated Principal-Agent Problem," Journal of Economic Theory 51, (1990), pages 367-390.

[31] Wang, Cheng. "Dynamic Insurance with Private Information and Balanced Budgets," Review of Economic Studies 62, (1995), pages 577-596.

[32] Zeldes, Steven. "Consumption and Liquidity Constraints: An Empirical Investigation," Journal of Political Economy 97, (1989), pages 305-346. 